\title{
EVALUACIÓN INICIAL DE UN ENSAYO DE MANEJO EN Acacia dealbata
}

\author{
Juan Carlos Pinilla $S^{1}$. y Mauricio Navarrete $\mathrm{T}^{2}$.
}

\section{RESUMEN}

Dentro de su programa de investigación sobre acacias, que busca posicionar el uso de estas especies y su utilización económica en el país, el Instituto Forestal ha desarrollado diversos trabajos y experiencias orientadas al manejo silvícola de diferentes especies del género Acacia, con el fin de generar una base experimental para su uso como alternativas a las tradicionalmente empleadas en la forestación en diversas áreas de la zona central y sur del país.

Se está trabajando principalmente con Acacia saligna en la zona semiárida, y con Acacia dealbata, Acacia mearnsii y Acacia melanoxylon, en la zona centro sur, especies que presentan buenos crecimientos y ofrecen productos alternativos y complementarios a aquellos de las especies habituales de las plantaciones del país, permitiendo además utilizar áreas y suelos que resultan marginales para el desarrollo de estas.

Entre las experiencias realizadas se encuentra un ensayo de raleos establecido en la Región del Maule con Acacia dealbata cuyos resultados iniciales se presentan en este trabajo. Se trata de una plantación efectuada en el año 2005, que fue sometida a raleos en el año 2008 y los resultados preliminares evaluados en el año 2010.

Palabras clave: Acacia dealbata, manejo, raleos.

\section{SUMMARY}

The Chilean forest Institute is carrying out a research program on Acacia species, which main objective is to generate an experimental basis to support the development of forest plantations by using those species as an alternative to the traditional ones in different zones of the country.

The research program includes Acacia saligna, in the semiarid zone, and Acacia dealbata Acacia mearnsii and Acacia melanoxylon, in the central zone, fast growing species that can offer not only alternative and complementary products to those of the usual species in afforestation in the country, but also can be used in areas and soils that are marginal sites for the growth of the most used species.

Among the various experiences, a thinning trial with Acacia dealbata was established in the Región del Maule and the preliminary results are presented in this paper. The plantation was installed in 2005, the thinning treatments were performed in 2008 and the preliminary evaluation in 2010.

Keywords: Acacia dealbata, silviculture, thinning. 


\section{INTRODUCCIÓN}

Una importante y permanente línea de investigación del Instituto Forestal corresponde al establecimiento, manejo, protección y utilización de plantaciones forestales, trabajos que se iniciaron en 1962 con el programa de introducción de especies forestales, que tenía por objetivo principal presentar nuevas alternativas productivas, en especial para aquellas áreas donde las plantaciones con especies tradicionales como pino y eucalipto no son posibles o son de baja rentabilidad, debido a condiciones de sitio marginales para su crecimiento y desarrollo.

De los avances y resultados de ese programa inicial se han derivado múltiples investigaciones posteriores sobre diferentes especies, técnicas de vivero y propagación, métodos de establecimiento de plantaciones, manejo silvícola, mejoramiento genético, utilización y otras, orientadas a especies de rápido crecimiento y variedad de usos.

Entre las especies que han resultado de interés se encuentra algunas del género Acacia, que cuenta con especies forestales de interés comercial, cuyas maderas presentan usos alternativos y complementarios a aquellos de las especies tradicionales, agregándose a esto turnos de cosecha reducidos (khanna, 1999; Pinilla, 2000; Barros, 2007). Entre ellas se encuentran Acacia dealbata, Acacia mearnsii y Acacia melnoxylon, que por su rápido crecimiento en el país y su variedad de usos, dieron origen al programa de investigación sobre acacias que desarrolla el Instituto Forestal con el apoyo de fondos concursables de Innova CORFO.

Este programa de investigación abarca desde la selección de procedencias de semillas, la propagación, el establecimiento de plantaciones, el mejoramiento genético y el manejo, hasta la utilización para diferentes fines industriales, como pulpa y papel, aserrío, tableros, energía, productos químicos y otros (Pinilla et al., 2010 y Hernández y Pinilla, 2010).

La información reunida hasta ahora indica que las acacias en estudio son especies promisorias por su crecimiento y sus posibilidades industriales. Los resultados que se están obteniendo en los diferentes ensayos, respecto de su crecimiento, propiedades y aptitudes de sus maderas, entregan buenas perspectivas potenciales para estas especies.

Sin embargo, la obtención de productos de mayor valor exige la aplicación de silvicultura intensiva y superficies suficientes de plantaciones para ubicar a estas especies como alternativas interesantes y complementarias a las especies tradicionales. La experiencia ganada en Nueva Zelandia así lo indica.

INFOR ha avanzado en materia de técnicas de establecimiento de plantaciones, selección de procedencias, estudios de crecimiento en plantaciones originadas de semilla local y australiana, y está dando los primeros pasos para un programa de mejoramiento genético mediante ensayos procedencia/progenie e investigaciones sobre propagación gámica y agámica. Igualmente ha realizado estudios sobre las propiedades y aptitudes de uso de la madera y ha iniciado estudios para validar esquemas de manejo silvícola en diferentes sitios y en función de distintos productos.

Los esquemas de manejo silvícola deben contemplar básicamente secuencias de raleos, que buscan concentrar el crecimiento en un menor número de ejemplares, alcanzando 
mayores diámetros, y secuencias de podas, que sucesivamente van limpiando el fuste de ramas en altura, con el fin de generar la mayor proporción posible de su volumen libre de nudos.

Estos esquemas permiten al propietario obtener algunos productos intermedios de los raleos y madera de mayor valor en la cosecha, en tanto que el procesamiento industrial posterior de la madera generará también productos de mayor valor.

Para producir madera de alta calidad se deben tener como objetivo las trozas para foliado, que corresponden a la mejor calidad, cuyas dimensiones mínimas actualmente corresponden a $3 \mathrm{~m}$ de largo y $40 \mathrm{~cm}$ de diámetro, con un cilindro central nudoso de máximo 10 $-12 \mathrm{~cm}$. El largo mínimo de troza es 2,20 - 2,50 m, pero si es mayor el valor de la troza aumenta en forma más que proporcional y algo similar ocurre con el diámetro.

Sin embargo, en la práctica no es raro encontrar trozas de excelentes dimensiones con decoloraciones, manchas negras o defectos internos que las deprecian fuertemente. Parte importante de esos defectos se origina en técnicas de poda inapropiadas o la ausencia de ellas. Conviene considerar que la poda es una técnica imprescindible cuando se espera ingresar al mercado de la madera de calidad y alto valor, y que los costos a ella asociados corresponden a una inversión.

Cada árbol debe observarse desde todos los ángulos, para tener una apreciación global de su situación, la observación desde sólo un punto induce serios errores en la poda. Luego, se establece el tipo de dominancia apical del individuo (elevada, media, baja o nula), ya que cada categoría requiere de una poda diferente. Si un individuo no presenta dominancia apical marcada, la poda de formación es necesaria para asegurar la formación de troncos rectos sin nudos (Crawford, 1996).

Se define el ápice y eliminan las ramas según el tipo de poda seleccionada, privilegiando la rectitud del eje y el balance del árbol. Para asegurar la buena cicatrización y evitar la entrada de patógenos por la herida de poda, esta se debe realizar cuidando que el corte se realice tan cerca como sea posible al cuello de la rama. La forma adecuada es comenzar por eliminar las ramas gruesas y mal ubicadas, evitando el desgarramiento de la corteza. No deben quedar muñones y nunca hay que eliminar el cuello o anillo cicatricial, ni cortar por detrás de la arruga de la corteza de la rama (Shigo, 1994).

Para la producción de madera de la mejor calidad bajo esquemas de arboricultura o manejo intensivo, en particular para producir acacias de calidad, se recomienda investigar los efectos de una poda de formación seguida de podas paulatinas de levante sobre la calidad y crecimiento del árbol.

INFOR ha instalado ensayos de manejo en Acacia dealbata con el objetivo de determinar esquemas de intervención de podas y raleos que permitan obtener madera de adecuada calidad para los productos industriales que se considera que esta especie puede entregar.

Entre estos productos destacan madera aserrada de gran trabajabilidad y facilidad de secado, con un atractivo color y veteado, y chapas, que presentan interesantes características de calidad (Briones y Pinilla, 2006). Para obtener madera apropiada para estos productos se 
requiere de las intervenciones de manejo mencionadas (Pinilla et al., 2006).

Dado que los ensayos realizados son recientes, no existe aún información local sobre esquemas de manejo de acacias. De investigaciones desarrolladas en Nueva Zelanda con Acacia melanoxylon (Blackwood en Australia y Nueva Zelanda, aromo australiano en Chile) se reportan ensayos de manejo silvícola que inicialmente utilizaron un mínimo de 450 arb/ha hasta un máximo de 2.550 arb/ha, junto con ensayos de poda.

Concluyen en la necesidad de dejar siempre una copa viva de $3 \mathrm{~m}$ para una cosecha final de $200 \mathrm{arb} / \mathrm{ha}$ y desarrollar propuestas de esquemas de manejo para la especie como la que indica el Cuadro $N^{\circ} 1$ (Nicholas and Brown, 2002).

\section{Cuadro $N^{\circ} 1$}

\section{PROPUESTA SILVÍCOLA PARA Acacia melanoxylon}

(Fuente: Nicholas and Brown, 2002)

\begin{tabular}{|c|c|c|c|c|c|c|c|}
\hline \multirow{2}{*}{$\begin{array}{l}\text { Edad } \\
\text { (Años) }\end{array}$} & \multirow{2}{*}{$\begin{array}{c}\text { Densidad } \\
\text { (arb/ha) }\end{array}$} & \multirow{2}{*}{$\begin{array}{l}\text { DAP } \\
(\mathrm{cm})\end{array}$} & \multirow{2}{*}{$\begin{array}{c}\text { Altura } \\
(\mathrm{m})\end{array}$} & \multicolumn{3}{|c|}{ Volumen } & \multirow[t]{2}{*}{ Observaciones } \\
\hline & & & & $\begin{array}{l}\text { Unitario } \\
\left.\text { ( } \mathrm{m}^{3} / \mathrm{arb}\right) \\
\end{array}$ & $\begin{array}{c}\text { Total } \\
\left(\mathrm{m}^{3} / \mathrm{ha}\right)\end{array}$ & $\begin{array}{c}\text { A Extraer } \\
\left(\mathrm{m}^{3} / \mathrm{ha}\right)\end{array}$ & \\
\hline 1 & 1600 & 1,2 & 0,9 & 0,006 & 9,19 & & Plantacion a 2,5 × 2,5 m \\
\hline 2 & 1600 & 2,4 & 1,8 & 0,006 & 9,60 & & \\
\hline 7 & 1600 & 8,4 & 6,3 & 0,018 & 29,56 & & \\
\hline 8 & 1350 & 9,6 & 7,2 & 0,025 & 33,44 & 6,19 & Primer raleo \\
\hline 9 & 1350 & 10,8 & 8,1 & 0,033 & 44,35 & & \\
\hline 11 & 1350 & 13,3 & 10,0 & 0,057 & 76,37 & & Primera poda a $4 \mathrm{~m}$ \\
\hline 15 & 1100 & 18,5 & 14,0 & 0,144 & 158,13 & 35,94 & Segundo raleo \\
\hline 16 & 1100 & 19,8 & 15,0 & 0,175 & 192,71 & & Segunda poda a $7,5 \mathrm{~m}$ \\
\hline 22 & 850 & 26,8 & 20,4 & 0,429 & 364,30 & 107,15 & Tercer raleo \\
\hline 23 & 850 & 27,7 & 21,1 & 0,473 & 402,11 & & \\
\hline 29 & 600 & 33,0 & 25,3 & 0,803 & 481,55 & 200,64 & Cuarto Raleo \\
\hline 30 & 600 & 34,0 & 26,0 & 0,874 & 524,61 & & \\
\hline 36 & 350 & 38,2 & 29,0 & 1,226 & 429,14 & 306,53 & Quinto raleo \\
\hline 37 & 350 & 38,9 & 29,5 & 1,293 & 452,43 & & \\
\hline 41 & & 41,7 & 31,5 & 1,583 & & 554,05 & Cosecha \\
\hline
\end{tabular}

(Fuente: Nicholas and Brown, 2002)

Sobre la madera de Acacia melanoxylon en Nueva Zelandia, se ha concluido que el sitio y la silvicultura pueden influir en su densidad y que las tasas de crecimiento tienen influencia sobre esta dado que la densidad básica de la madera disminuye a mayor velocidad de crecimiento. El porcentaje de madera dura disminuye con un crecimiento más rápido y la tasa de crecimiento tendría una influencia pequeña sobre el color de la madera (Pinilla, 2000). 
En Nueva Zelandia se recomienda una serie de prácticas al momento de utilizar la especie, entre las cuales se destaca el establecer rodales de poca extensión utilizando semilla común, aplicación de esquemas de raleos y podas estándar y una cosecha final al año 35 . Se destaca como innecesario el uso de árboles acompañantes para obtener una buena forma en el árbol3.

Las conclusiones del trabajo desarrollado en Nueva Zelandia destacan la importancia del sitio en el desarrollo de Blackwood y de una silvicultura basada en podas y raleos.

En el caso de Acacia dealbata, no han desarrollado este tipo de propuesta, pero se señala que existe la posibilidad de desarrollo de productos aserrables de esta especie con esquemas de manejo con podas y raleos, los que requieren de mayor investigación.

Acacia dealbata, Silver Wattle, originaria del SE de Australia, comúnmente llamada aromo del país en Chile, pese a su origen australiano, es una especie de muy rápido crecimiento que ha respondido en forma muy interesante a las condiciones edafoclimáticas locales y genera una madera adecuada para una variedad de usos.

El presente trabajo presenta los primeros resultados de un ensayo de raleos efectuado con esta especie en la Región del Maule.

\section{OBJETIVOS}

\section{Objetivo General del Programa}

Escalar las opciones productivas del recurso forestal compuesto por las especies Acacia mearnsii, A. dealbata y A. melanoxylon a través del fomento, masificación y desarrollo de productos con mayor valor agregado

\section{Objetivo Específico del Ensayo}

Entregar los primeros antecedentes que permitan obtener madera de mayor valor y promover el desarrollo de herramientas de apoyo para el manejo y cultivo de Acacia dealbata en Chile.

\section{METODOLOGÍA}

\section{Antecedentes Generales del Sitio de Ensayo}

Para la instalación del ensayo de raleos se utilizó unidad demostrativa previamente plantada, que tenía por objeto transferir en forma práctica las experiencias sobre el comportamiento de Acacia en diversos sitios.

Esta unidad fue establecida durante el año 2005 en el fundo Copihue, perteneciente a la Compañía Agrícola y Forestal El Álamo, en la Comuna de Retito, Región del Maule.

3 Ian Nicholas, 2006. Ensis Genetics, The Join Forces of CSIRO and SCION, Comunicación personal. 
El sector seleccionado corresponde a suelos planos, cuyo uso anterior había sido plantaciones de eucalipto, rodeados de plantaciones de álamo de la empresa.

La unidad tiene una superficie de 1 ha plantada con Acacia dealbata. Se utilizaron plantas producidas en contenedores individuales de $100 \mathrm{~cm}^{3}$ de capacidad. Las semillas utilizadas en la unidad son de origen australiano y corresponden a dos procedencias de Tasmania (16385 SSE Snug TAS y 16384 S Orford TAS).

La plantación se realizó en Octubre de 2005 con un espaciamiento de 2 × 3 metros, es decir con una densidad inicial nominal de 1.667 plantas por hectárea.

La preparación previa del sitio contempló un subsolado a una distancia de 3 metros entre surcos y una profundidad de $70 \mathrm{~cm}$, y mullido de la zona de plantación cada 2 metros.

Durante la plantación se aplicó 2 gramos de gel. Posterior a la plantación y dada la fecha de ella, se aplicó un riego utilizando los canales existentes en el sector, junto con un desmalezado.

En la Figura $N^{\circ} 1$ se muestra el sitio de plantación, el subsolado, la plantación y el riego, y en la Figura $N^{\circ} 2$ se muestra un aspecto general de la plantación en el año 2007 cuando tenía dos años de edad. 

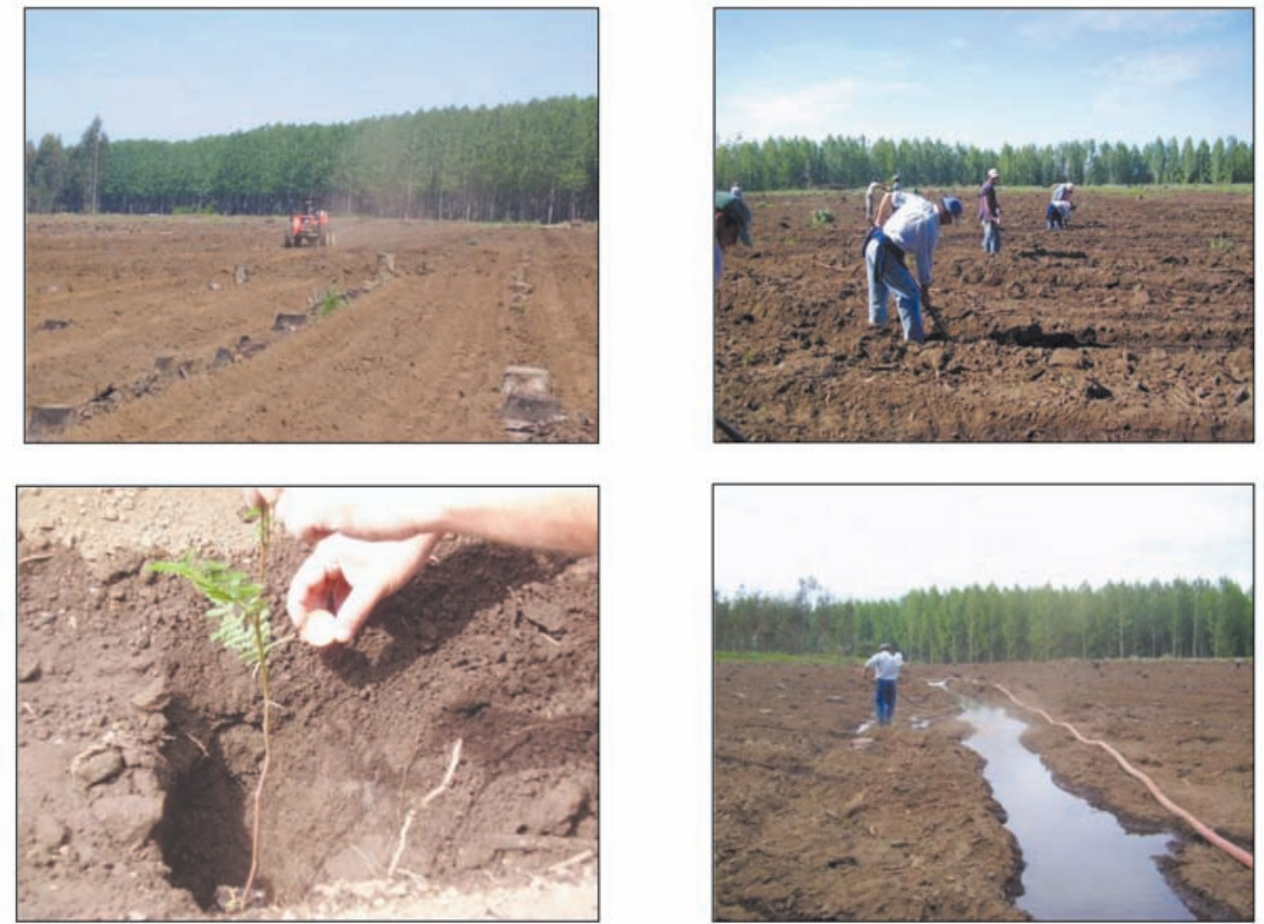

Figura $N^{\circ} 1$

ESTABLECIMIENTO DE LA PLANTACIÓN
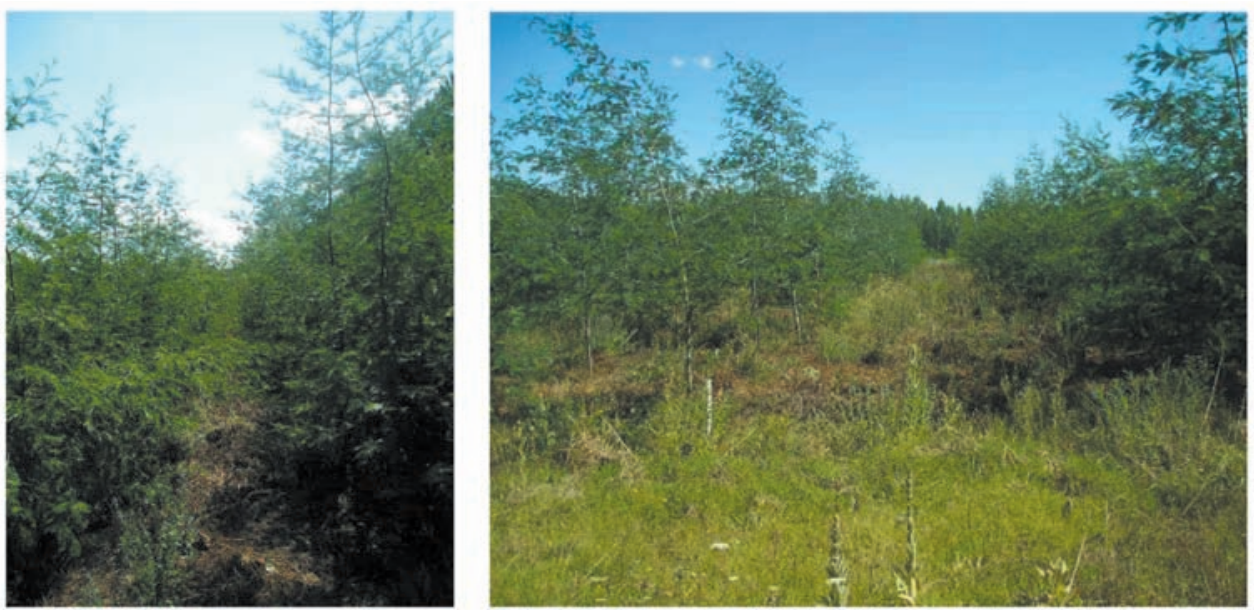

Figura $\mathrm{N}^{\circ} 2$

VISTA GENERAL DE LA PLANTACIÓN EN 2007 


\section{Antecedentes del Ensayo de Poda y Raleo}

En las plantaciones ya descritas se instaló el ensayo de raleos en el año 2008, es decir cuando las plantaciones tenían 3 años de edad.

\section{Diseño del Ensayo}

El diseño experimental es de parcelas al azar con tres repeticiones, considerando tres tratamientos; 2 regímenes de raleos y un testigo sin raleos (Figura $\mathrm{N}^{\circ} 3$ ).

El primer raleo se aplicó el año 2008 cuando el rodal contaba con 3 años de edad.

Se aplicó además un régimen de poda basado en conseguir una troza libre de nudos de al menos 4 metros generada a través de los 2 raleos y cuya altura de poda dependerá de la altura de los árboles.

Se ensayan tratamientos de raleo de mayor a menor intensidad, pero en todos los casos se contempla llegar a densidad final de 300 a 400 arb/ha (Cuadro № 2).

\begin{tabular}{|c|c|c|c|c|}
\cline { 2 - 5 } \multicolumn{1}{c|}{} & T1 & T1 & T1 & T2 \\
\hline T3 & T3 & T3 & T2 & T2 \\
\hline & & & & \\
\hline & & Camino & & \\
\hline
\end{tabular}

Figura $\mathrm{N}^{\circ} 3$

DISEÑO DEL ENSAYO

Cuadro $\mathrm{N}^{\circ} 2$

TRATAMIENTOS DEL ENSAYO

\begin{tabular}{|c|c|c|c|c|}
\hline TRATAMIENTO & $\begin{array}{l}\text { DENSIDAD } \\
\text { INICIAL } \\
\text { (arb/ha)* }\end{array}$ & 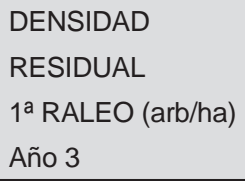 & 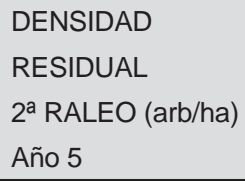 & $\begin{array}{l}\text { DENSIDAD } \\
\text { RESIDUAL } \\
\text { 3a RALEO (arb/ha) } \\
\text { Año } 8\end{array}$ \\
\hline T1 (Testigo) & 1.727 & 1.727 & 1.727 & 1.727 \\
\hline T2 (R1) - 25\% & 1.727 & 1.360 & 800 & 400 \\
\hline T3 (R2) - 50\% & 1.727 & 1.047 & 400 & \\
\hline
\end{tabular}

*: Densidad real observada en terreno 


\section{- Variables a Medir}

Anualmente se medirán las siguientes variables:

Supervivencia (\%)

$\operatorname{DAP}(\mathrm{cm})$

Altura total $(\mathrm{m})$

Estado del árbol (según parámetros de sanidad y forma)

También se evaluará luego de 2 años de aplicado el raleo final:

Altura Comienzo copa $(\mathrm{m})$

Diámetro de inicio de copa $(\mathrm{cm})$

Diámetro de copa: Medición 4 radios (N, S, E y O) (a una submuestra de árboles) (m)

Factor de forma (ej. diámetro a un tercio de la altura del árbol u otra variable que permita estimar forma).

La medición será anual durante el periodo de ejecución del proyecto, continuándose con mediciones bianuales hasta que el rodal tenga 10 años de edad, para luego continuar las mediciones a los 13 y 15 años.

Dado los objetivos de estos ensayos, en donde los efectos de los esquemas de manejo planteados se reflejan hasta 2 años después de su aplicación, es necesario mantener las mediciones a fin de detectar el real efecto de este tipo de manejo sobre el crecimiento y la madera de estas plantaciones.

\section{- Planteamiento en Terreno del Ensayo}

Para la instalación del ensayo, previamente se recorrió la unidad con Acacia dealbata a fin de determinar en qué sector se instalaría.

Una vez seleccionada el área, se procedió a marcar en terreno la distribución del ensayo y la asignación de los tratamientos en cada una de las 9 parcelas Posteriormente, se procedió a intervenir cada parcela a fin de aplicar el tratamiento asignado en cada una de ellas.

Posteriormente se procedió a la marcación, medición de todos los árboles y ejecución del raleo de los árboles que corresponda según tratamiento, compatibilizando la selección de los mejores individuos y una distribución regular de ellos en terreno.

Los árboles residuales quedaron claramente identificados (numerados). Posteriormente se aplicó una poda, y se tomaron las medidas necesarias para minimizar riesgos de daños bióticos y abióticos, si estos son probables. En la Figura $\mathrm{N}^{\circ} 3$ se puede apreciar las actividades de instalación del ensayo. 

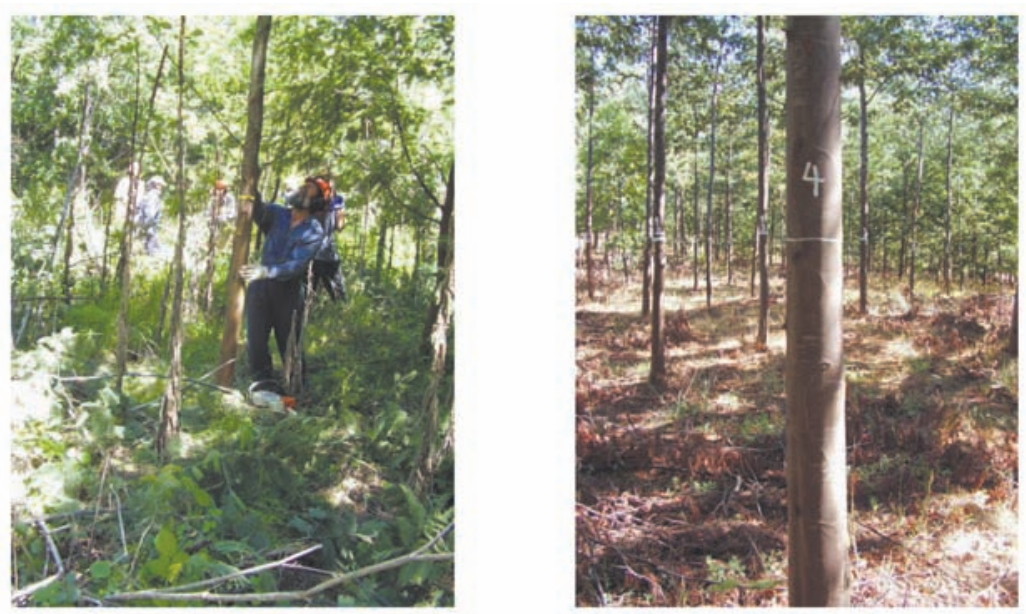

Figura $\mathrm{N}^{\circ} 3$

INSTALACIÓN ENSAYO RALEOS

\section{RESULTADOS}

\section{Estado del Rodal Previo a la Instalación del Ensayo}

En el mes de marzo del año 2008 se realizó la primera evaluación del rodal, previo a la instalación del ensayo de manejo. Para ello se instaló al azar una parcela temporal de 500 $\mathrm{m}^{2}$, en la cual se midieron todos los diámetros y una muestra de alturas. Posteriormente la información fue procesada y permitió generar las variables de rodal más relevantes para esta unidad. Los valores obtenidos se presentan en el Cuadro $\mathrm{N}^{\circ} 3$ y Figura $\mathrm{N}^{\circ} 4$.

\section{Cuadro $\mathrm{N}^{\circ} 3$ VARIABLES DE RODAL}

\begin{tabular}{|l|l|l|l|l|l|l|l|}
\hline PARCELA & $\begin{array}{c}\text { EDAD } \\
(\mathbf{a n ̃ o s})\end{array}$ & $\begin{array}{c}\text { ALTURA } \\
\text { MEDIA } \\
(\mathbf{m})\end{array}$ & $\begin{array}{c}\text { DIÁMETRO } \\
\text { MEDIO } \\
(\mathbf{c m})\end{array}$ & $\begin{array}{c}\text { VOLUMEN } \\
\left(\mathbf{m}^{3} / \mathbf{h a}\right)\end{array}$ & $\begin{array}{c}\text { ÁREA } \\
\text { BASAL } \\
\left(\mathbf{m}^{2} / \mathrm{ha}\right)\end{array}$ & $\begin{array}{c}\text { ALTURA } \\
\text { DOMINANTE } \\
(\mathbf{m})\end{array}$ & $\begin{array}{c}\text { DENSIDAD } \\
(\mathbf{a r b} / \mathrm{ha})\end{array}$ \\
\hline 106 & 2,65 & 8,0 & 7,0 & 28,14 & 5,69 & 9,8 & 1.380 \\
\hline
\end{tabular}




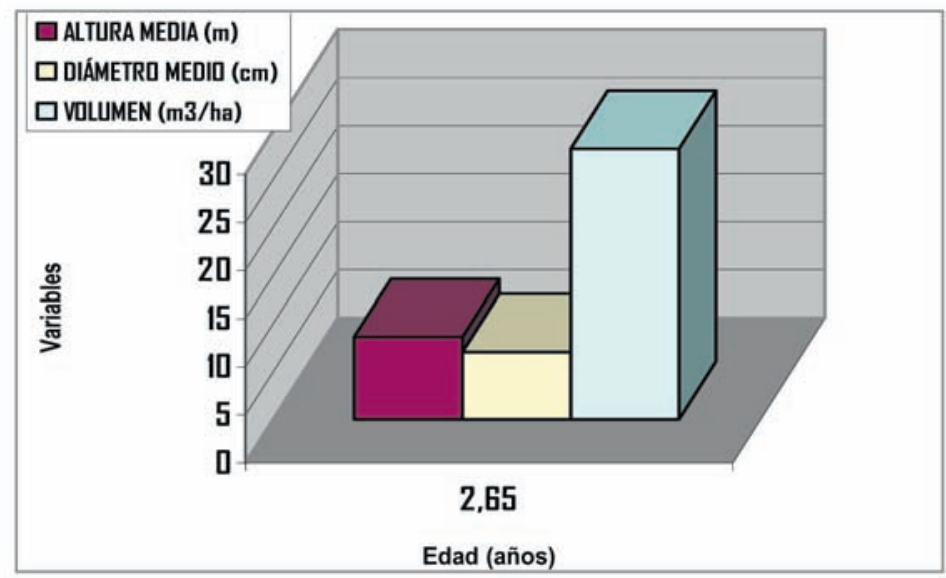

Figura $N^{\circ} 4$

VARIABLES DE RODAL

(Edad 2,6 años)

Los valores registrados en la unidad son interesantes y demuestran el potencial de crecimiento de la especie. Es así como los $28 \mathrm{~m} 3 /$ ha obtenidos en volumen representan un incremento medio anual cercano a los $10 \mathrm{~m}^{3}$.

\section{- Resultados de la Primera Evaluación del Ensayo}

En el mes de Abril del año 2010 se realizó la primera evaluación del rodal luego de instalado el ensayo de manejo.

Para ello se controlaron las 9 parcelas que constituyen el ensayo, midiendo en cada una todos los diámetros y una muestra de alturas. Posteriormente, la información fue procesada y se obtuvieron las variables agregadas descriptoras más representativas de cada tratamiento aplicado.

\section{- Evolución del Rodal}

Se presenta la comparación de las variables más representativas entre la situación del año 2008 y los promedios generales obtenidos el año 2010 (Figuras $\mathrm{N}^{\circ} 5$ y Nº). 


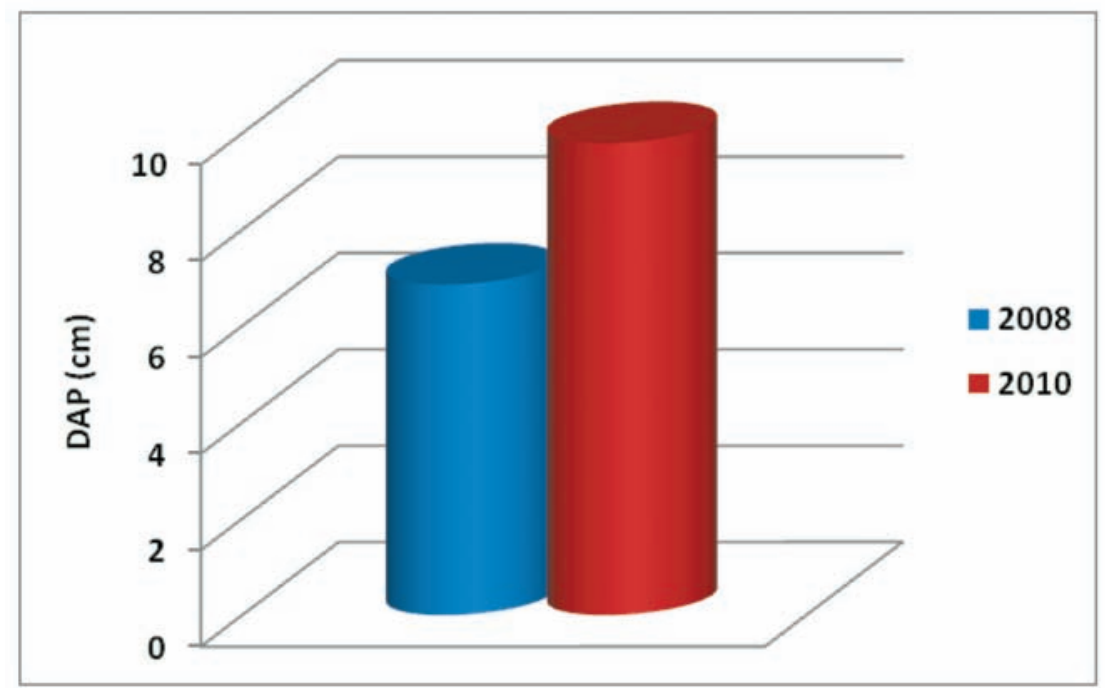

Figura $\mathrm{N}^{\circ} 5$

DIAMETRO MEDIO RODAL 2008 Y 2010

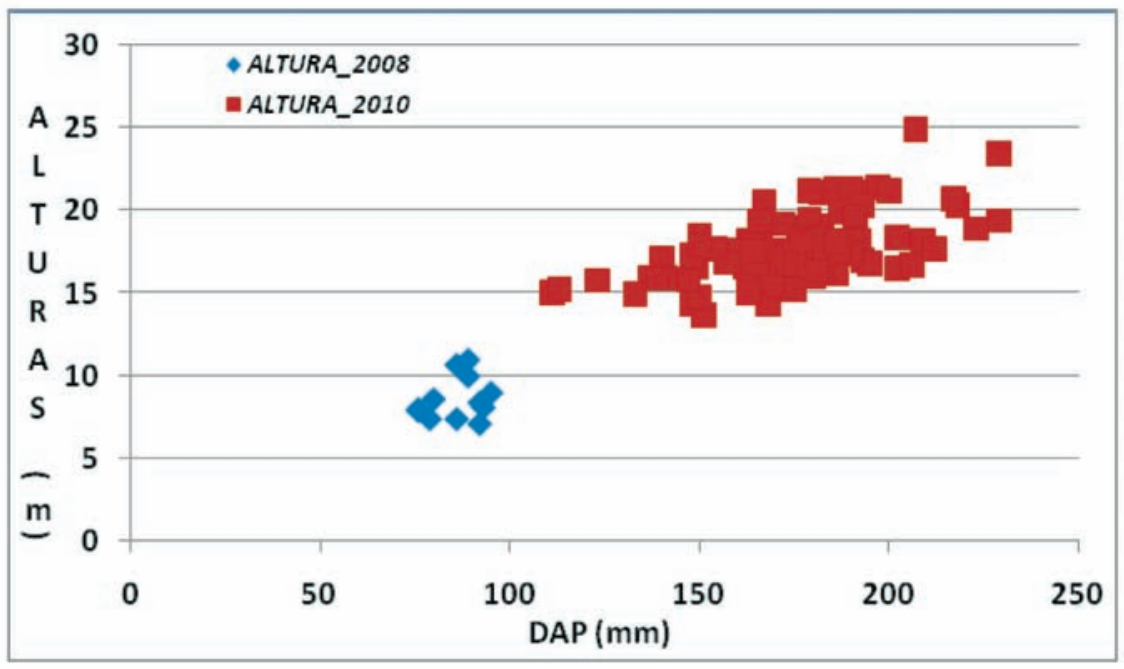

Figura $N^{\circ} 6$

ALTURA MEDIA RODAL 2008 Y 2010

Se puede apreciar un interesante desarrollo del rodal entre las edades 2,6 y 4,8 años, observándose alturas de hasta $25 \mathrm{~m}$. 


\section{- Evaluación Inicial del Ensayo de Raleo}

Los tratamientos evaluados en la medición del año 2010 corresponden a la primera etapa del esquema de manejo a analizar (Cuadro $\mathrm{N}^{\circ} 4$ ).

Cuadro $\mathrm{N}^{\circ} 4$

\section{ESQUEMAS DE MANEJO}

\begin{tabular}{|c|c|c|}
\hline TRATAMIENTO & $\begin{array}{c}\text { DENSIDAD RESIDUAL } \\
\mathbf{1}^{\circ} \text { RALEO Año 3 (2008) } \\
(\%)\end{array}$ & $\begin{array}{c}\text { DENSIDAD RESIDUAL REAL } \\
\mathbf{1}^{\circ} \text { RALEO (arb/ha) } \\
\text { Año 3 (2008) }\end{array}$ \\
\hline TESTIGO - T1 & $100 \%$ & 1.727 \\
\hline T2 & $75 \%$ residual & 1.360 \\
\hline T3 & $50 \%$ residual & 1.047 \\
\hline
\end{tabular}

Los resultados obtenidos en evaluación 2010, a los aproximadamente 5 años de edad, a nivel de las variables agregadas más relevantes, se presentan en el Cuadro $N^{0} 5$ y Figuras $\mathrm{N}^{\circ} 7 \mathrm{a} \mathrm{N}^{\circ} 9$.

\section{Cuadro $\mathrm{N}^{\circ} 5$}

VARIABLES AGREGADAS ENSAYO SEGÚN TRATAMIENTO

\begin{tabular}{|c|c|c|c|c|c|c|c|c|}
\hline TRATAMIENTO & $\begin{array}{c}\text { DENSIDAD } \\
\text { RESIDUAL } \\
1^{\circ} \text { RALEO } \\
\text { Año } 3 \text { (2008) } \\
\text { (arb/ha) }\end{array}$ & $\begin{array}{c}\text { ALTURA } \\
\text { MEDIA } \\
\text { (m) }\end{array}$ & $\begin{array}{c}\text { DAP } \\
\text { MEDIO } \\
(\mathrm{mm})\end{array}$ & $\begin{array}{c}\text { VOLUMEN } \\
\left(\mathrm{m}^{3} / \mathrm{ha}\right)\end{array}$ & $\begin{array}{c}\text { Área } \\
\text { Basal } \\
\left(\mathrm{m}^{2} / \mathrm{ha}\right)\end{array}$ & $\begin{array}{c}\text { ALTURA } \\
\text { DOMI- } \\
\text { NANTE } \\
(\mathrm{m})\end{array}$ & $\begin{array}{l}\text { DENSI- } \\
\text { DAD RE- } \\
\text { SIDUAL } \\
\text { REAL } \\
\text { (arb/ha) }\end{array}$ & $\begin{array}{l}\text { EDAD } \\
\text { (años) }\end{array}$ \\
\hline TESTIGO - T1 & $100 \%$ & 14,8 & 124,8 & 135,889 & 23,33 & 17,4 & 1.727 & 4,8 \\
\hline T2 & $75 \%$ & 15,9 & 146,2 & 187,182 & 24,01 & 18,6 & 1.360 & 4,8 \\
\hline T3 & $50 \%$ & 17,6 & 165,9 & 157,492 & 23,44 & 20,5 & 1.047 & 4,8 \\
\hline
\end{tabular}




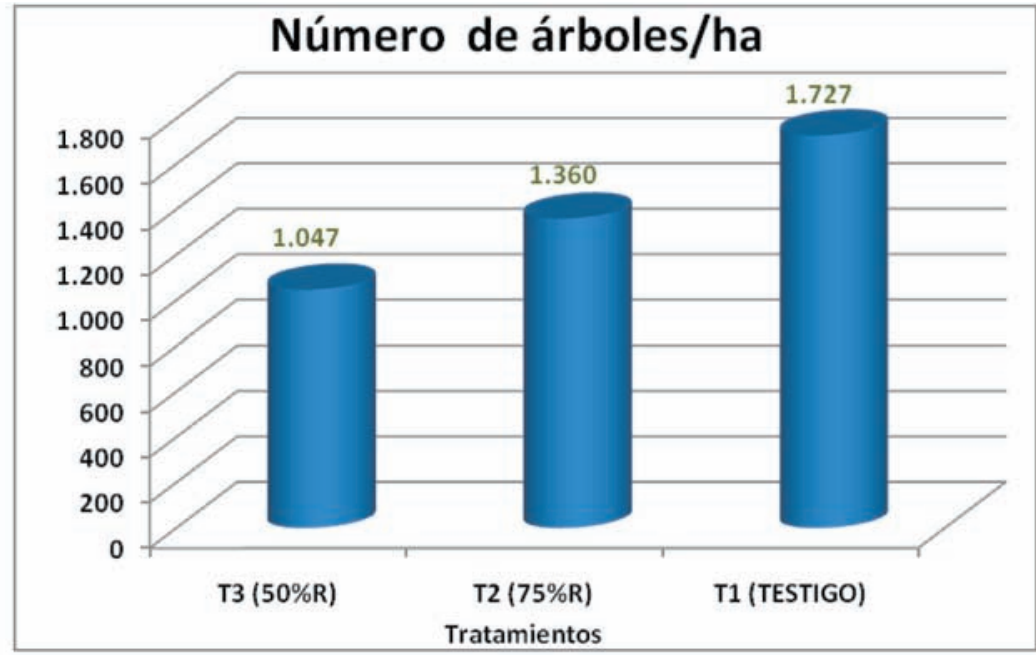

Figura $N^{\circ} 7$

DENSIDAD SEGÚN TRATAMIENTO

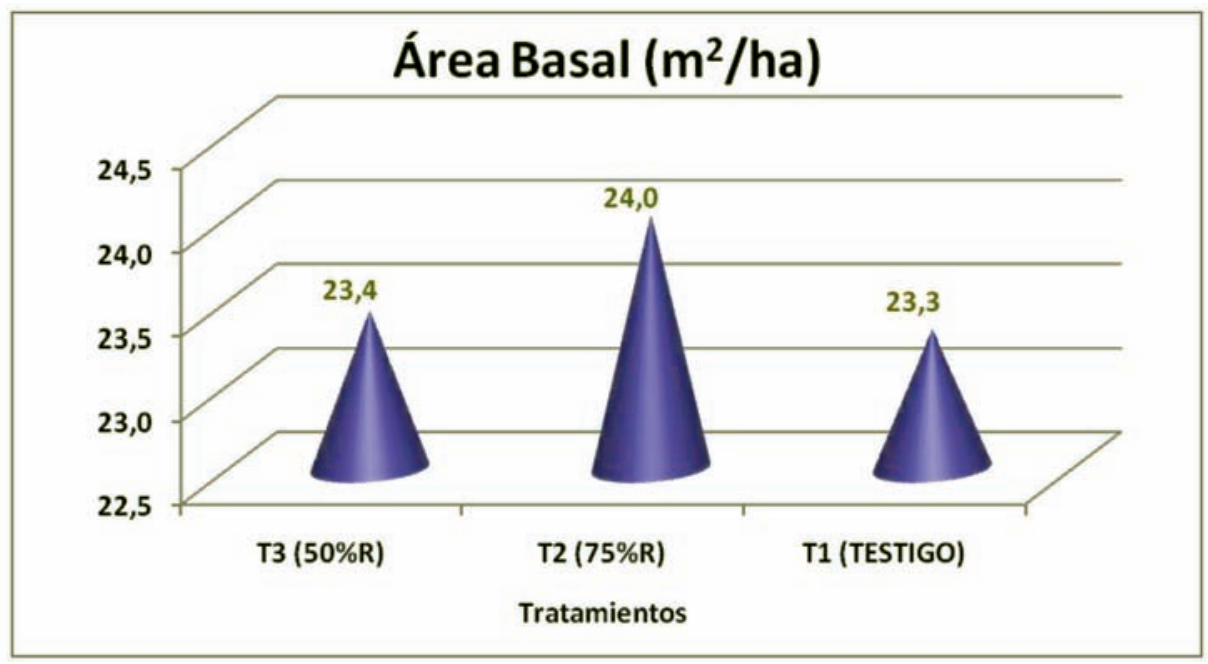

Figura $\mathrm{N}^{\circ} 8$

AREA BASAL SEGÚN TRATAMIENTO 


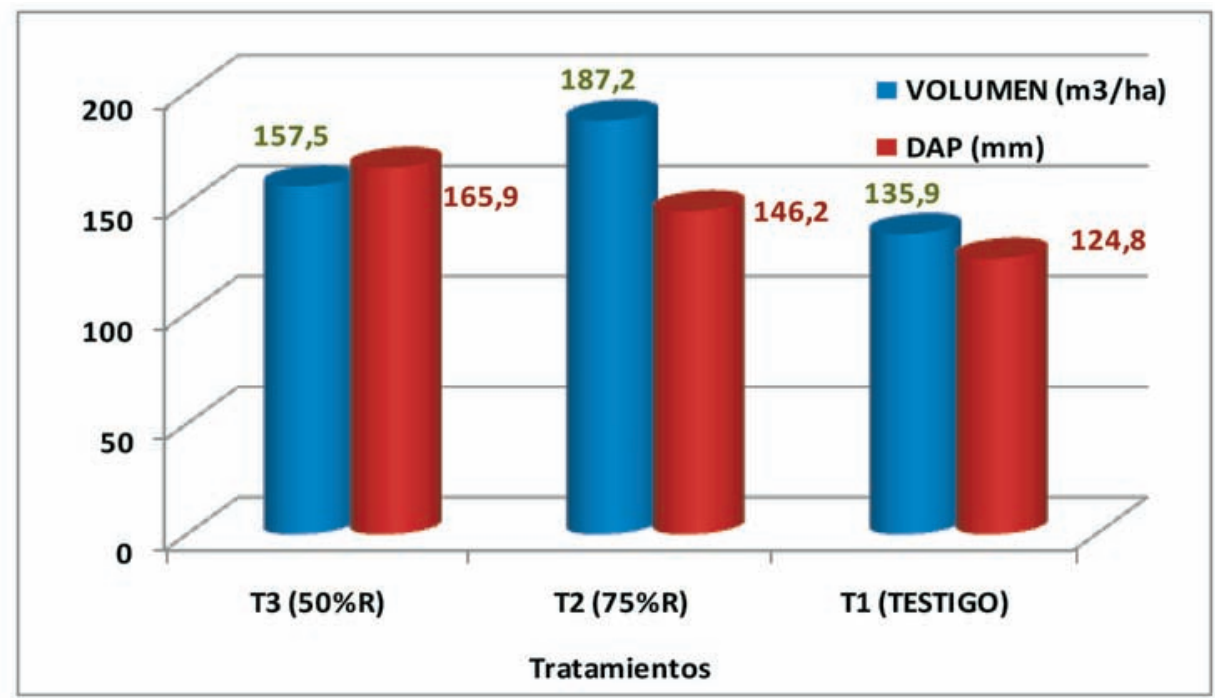

\section{Figura $N^{\circ} 9$. \\ VOLÚMENES Y DIÁMETROS MEDIOS SEGÚN TRATAMIENTO}

En primer lugar hay que señalar que el número de árboles residuales en el caso de los 2 tratamientos con raleo, no es el nominalmente estimado, siendo la situación real un número algo mayor al inicialmente programado debido a que la situación en terreno no permitió extraer el total de los árboles necesarios. Esta situación se corregirá en la segunda intervención del ensayo.

En los resultados se observa que el tratamiento $\mathrm{T} 2$, que considera aplicar en una primera etapa la extracción nominal cercana al 25\% del número de árboles, ha entregado los mejores resultados hasta el momento en términos del volumen total.

En relación el DAP promedio, el mayor valor se registra en el tratamiento con el menor número de árboles, lo que es coincidente con otros estudios similares en eucalipto o pino.

Hasta el momento es posible detectar diferencias estadísticamente significativas a nivel de DAP entre los 3 tratamientos (Cuadros $N^{\circ} 6$ y $N^{\circ} 7$ ), sucediendo algo similar con la altura media, lo que es necesario confirmar en futuras evaluaciones. 


\section{Cuadro $\mathrm{N}^{\circ} 6$}

ANÁLISIS DE VARIANZA PARA EL DAP ENTRE LOS DIFERENTES TRATAMIENTOS

\begin{tabular}{|c|c|c|c|c|c|}
\hline $\begin{array}{c}\text { Fuente de } \\
\text { Variación }\end{array}$ & $\begin{array}{c}\text { Suma de } \\
\text { Cuadrados }\end{array}$ & $\begin{array}{c}\text { Grados de } \\
\text { Libertad }\end{array}$ & $\begin{array}{c}\text { Cuadrado } \\
\text { Medio }\end{array}$ & F & p-valor \\
\hline Tratamiento & 151900,17 & 2 & 75950,08 & 56,74 & $<0,0001$ \\
\hline Error & 825851,44 & 617 & 1338,50 & \multicolumn{2}{|c}{} \\
\hline Total & 977751,60 & 619 & \multicolumn{2}{|c}{} \\
\cline { 1 - 3 } & & &
\end{tabular}

\section{Cuadro $\mathrm{N}^{\circ} 7$}

TABLA DE COMPARACIÓN DE MEDIAS

\begin{tabular}{|c|c|c|}
\hline Tratamiento & Media $(\mathbf{m m})$ & Nivel de Significancia \\
\hline TESTIGO - T1 $(100 \%)$ & 124,8 & $\mathrm{~A}$ \\
\hline T2 $(75 \%$ residual $)$ & 146,2 & $\mathrm{~B}$ \\
\hline T3 $(50 \%$ residual $)$ & 165,9 & $\mathrm{C}$ \\
\hline
\end{tabular}

Letras iguales señalan que no existen diferencias

significativas $(p<=0,05)$

Si bien el tratamiento que implica ralear a los 3 años un 25\% nominal del número de árboles presenta actualmente el mejor rendimiento en volumen, este resultado no es estadísticamente diferente del tratamiento testigo y del tratamiento con un porcentaje de raleo mayor. Esta información se presenta en los Cuadros $\mathrm{N}^{\circ} 8$ y $\mathrm{N}^{\circ} 9$.

\section{Cuadro $\mathrm{N}^{\circ} 8$}

\section{ANÁLISIS DE VARIANZA PARA EL VOLUMEN ENTRE LOS DIFERENTES TRATAMIENTOS}

\begin{tabular}{|c|c|c|c|c|c|}
\hline $\begin{array}{c}\text { Fuente de } \\
\text { Variación }\end{array}$ & $\begin{array}{c}\text { Suma de } \\
\text { Cuadrados }\end{array}$ & $\begin{array}{c}\text { Grados de } \\
\text { Libertad }\end{array}$ & $\begin{array}{c}\text { Cuadrado } \\
\text { Medio }\end{array}$ & F & p-valor \\
\hline Tratamiento & 3979,17 & 2 & 1989,59 & 1,13 & 0,3845 \\
\hline Error & 10604,38 & 6 & 1767,40 & & \\
\hline Total & 14563,56 & 8 & \multicolumn{2}{|c}{} \\
\cline { 1 - 3 } & & & &
\end{tabular}




\section{Cuadro $\mathrm{N}^{\circ} 9$ \\ TABLA DE COMPARACIÓN DE MEDIAS}

\begin{tabular}{|c|c|c|}
\hline Tratamiento & Media (m3/ha) & Nivel de Significancia \\
\hline TESTIGO - T1 $(100 \%)$ & 135,89 & $\mathrm{~A}$ \\
\hline T2 $(75 \%$ residual) & 187,18 & $\mathrm{~A}$ \\
\hline T3 (50\% residual) & 157,49 & $\mathrm{~A}$ \\
\hline
\end{tabular}

Letras iguales señalan que no existen diferencias

significativas $(p<=0,05)$

\section{ANÁLISIS DE LOS RESULTADOS Y CONCLUSIONES}

El Área Basal (medida de la ocupación del sitio) obtenida fue relativamente semejante en todos los tratamientos. Es interesante el estudio de esta variable ya que es un indicador para una situación óptima dentro del manejo silvicultural, en la búsqueda de la adecuada combinación de máxima Área. Basal, mínimo número de árboles y máximos incremento anuales en diámetro. Este resultado debe ser ratificado en las evaluaciones posteriores, estimando que debería de variar una vez establecido plenamente el ensayo y su evolución posterior. Este óptimo de combinación entre número de árboles, Área Basal y máximo incremento depende, a su vez de los productos que se quieran generar de estos bosques. Esta combinación será diferente según sea para producir madera pulpable, aserrada o cualquier otro producto intermedio que se pretenda obtener.

Si el objetivo es producir madera pulpable en rotaciones cortas no cabe duda que los tratamientos con un menor espaciamiento son los más aconsejables, ya que en ellos se produce la máxima ocupación del sitio con un alto número de árboles, obteniéndose así la mayor producción de volumen total por hectárea. Por otra parte, si se desea obtener madera aserrada en rotaciones más largas será necesario lograr una adecuada relación entre un máximo incremento en diámetro y el número de árboles por superficie.

Es necesario profundizar el estudio de los esquemas de manejo y su relación con distintos tipos de volúmenes bajo diferentes escenarios, estableciendo al mismo tiempo su efecto sobre los productos que se puedan generar de estos tipos de bosques, sea en cuanto a tipo, calidad, largos, formas y cualquier otra variable de interés factible de analizar.

Otro aspecto que será analizado más adelante corresponde al estudio del efecto de las podas en la generación de madera de alta calidad. Cuando el ensayo esté en su fase final, se extraerán árboles para realizar los estudios de calidad de madera según esquema utilizado.

Con la información obtenida desde cada una de las parcelas a través del tiempo, será posible identificar el efecto que cada uno de los esquemas de manejo ha provocado en los árboles de la plantación. Este efecto se verá reflejado en el crecimiento diametral y volumétrico de cada parcela, además de los resultados sobre la calidad de los productos a obtener. Será posible entonces construir modelos de crecimiento y/o rendimiento para cada tratamiento en 
particular, los que servirán para futuras estimaciones y definición de esquemas de raleo más recomendados.

De los resultados iniciales del ensayo se puede concluir hasta el momento que:

El DAP presentó diferencias estadísticamente significativas entre los distintos tratamientos. El mayor valor se presentó en el Tratamiento 1 (50\% de extracción del número de árboles), mientras que el menor valor lo presenta hasta el momento el tratamiento Testigo. En general, se confirma que a una menor densidad se obtienen mayores DAP.

Los menores valores en altura media se observan en los tratamientos de menor espaciamiento (Testigo). Por el contrario, los mayores incrementos en altura lo presentan los tratamientos con mayores espaciamientos.

Actualmente el esquema de raleo del $25 \%$ de los árboles a los 3 años y raleo futuro presenta el mejor rendimiento en volumen, siendo este resultado no estadísticamente diferente del tratamiento testigo y del tratamiento con un porcentaje de raleo mayor.

\section{REFERENCIAS}

Barros, S., 2007. El Género Acacia, Especies Multipropósito. En: Revista Ciencia e Investigación Forestal, Instituto Forestal. Número Extraordinario. Silvicultura y Utilización de Especies del Género Acacia. Noviembre 2007.

Briones, R. y Pinilla, J. C., 2006. Procesos Industriales y Aplicaciones de Acacia en Chile. En: $2^{\circ}$ Congreso Latinoamericano IUFRO. Bosques: La Creciente Importancia de sus Funciones Ambientales, Sociales y Económicas. La Serena, Chile. 23 al 27 de octubre de 2006.

Crawford, M., 1996. Walnuts: Production and Culture. Agroforestry Research Trust. 28 p.

Hernández, G. y Pinilla, J. C., 2010. Compendio, Propiedades de la Madera de Especies Nativas y Exóticas en Chile. Instituto Forestal, Informe Técnico Nº 178.

Khanna, P.K., 1999. Gains from Planting Eucalypts and Acacias, Onwood: Research Updates from CSIRO Forestry and Forest Products. Summer No. 23. CSIRO Forestry and Forest Products, Canberra.

Nicholas, I. and Brown, I., 2002. Blackwood. A Handbook for Growers and End Users. Forest Research Bulletin No25. Forest Research. Rotorua, New Zeland. 95p.

Pinilla, J. C., 2000. Descripción y Antecedentes Básicos sobre Acacia dealbata, A. melanoxylon y A. mearnsii. Revisión bibliográfica. Santiago, Chile, INFOR-CORFO. Informe Técnico 147. 49p.

Pinilla S., J. C.; Molina, M. P.; Briones, R. y Hernández, G., 2006. Opciones de Productos a partir de la Madera de Acacia, y su Promoción. Antecedentes de una Experiencia con Acacias en Chile. Boletín Informativo CIDEU N² 2, $2^{\circ}$ Trimestre 2006, Huelva, España. 
Pinilla, J. C., Molina, M. P.; Hernández, G., Barros, S., Ortíz, O. y Navarrete, M., 2010. Avances de la Investigación con Especies del Género Acacia en Chile. Instituto Forestal, Informe Técnico $\mathrm{N}^{\circ} 179$.

Shigo, L. A., 1994. Arboricultura Moderna Compendio. Un Sistema para el Cuidado de los Árboles y sus Asociados. Dirham, New Hampshire, USA. 152p. 
